\title{
Multiple contemporary arterial dissection in Ehlers-Danlos syndrome type IV
}

\author{
Anna Rocci, ${ }^{1}$ Enrico Paganelli, ${ }^{1}$ Angelo Rossetti, ${ }^{1}$ Carla Marcato, ${ }^{2}$ Davide Martorana, ${ }^{3}$ Maria Ilaria Tassoni, \\ Roberto Quintavalla ${ }^{1}$ \\ ${ }^{1}$ Angiology Unit; ${ }^{2}$ Radiology Unit; ${ }^{3}$ Unit of Medical Genetics, Parma University Hospital, Parma, Italy
}

\begin{abstract}
We report a case of multiple spontaneous arteries dissection in a 52-year-old female; the patient had a relevant family history of vascular complications and typical features so we hypothesized vascular Ehlers-Danlos syndrome (EDS) that was confirmed by genetic analysis of $C O L 3 A 1$ gene. We adopted a conservative approach: the patient was treated with heparin in the acute phase followed by aspirin and then celiprolol was started on the basis of a recent trial that demonstrates a reduction in arterial events in EDS patient treated. A careful follow-up was done with Doppler ultrasound and computed tomography scan, as non-invasive diagnostic techniques are preferred in these patients, and no other vascular symptomatic events have occurred. We tested all living relatives: half of them had COL3A1 mutation, they were referred to another center specialized in rare diseases and EDS for long-term follow-up and genetic counseling. This case demonstrates as a careful evaluation of clinical signs, clinical history of the patient and his family has allowed a definitive diagnosis, proper management of the patient during the acute event and in terms of prophylaxis of recurrence.
\end{abstract}

\section{Introduction}

Ehlers-Danlos syndrome (EDS) is a group of inherited connective tissue disorders variably affecting skin, joint, hollow viscera and blood vessels caused by a defect in the synthesis of collagen. ${ }^{1}$ Vascular or type IV EDS is an autosomal dominant defect in type III collagen synthesis affecting approximately 1 in 100,000 to 250,000 people. ${ }^{2}$ Mutations in COL3A1 have been identified. Vascular EDS is considered one of the most serious forms of EDS because blood vessels and organs are prone to tearing. ${ }^{3}$

\section{Case Report}

A 52-year-old female was referred to our hospital for surgical correction of hallux valgus. In the postoperative

Correspondence: Anna Rocci, AOU Parma, via Gramsci 14 43125 Parma, Italy.

Tel.: +39.0521.702093 - Fax: $+39.0521 .703795-$

Mobile: +39.340 .406998 .

E-mail: arocci@ao.pr.it

Key words: Ehlers-Danlos syndrome, arterial dissection.

Received for publication: 25 February 2014.

Accepted for publication: 6 April 2014.

This work is licensed under a Creative Commons Attribution NonCommercial 3.0 License (CC BY-NC 3.0).

CC Copyright A. Rocci et al., 2015

Licensee PAGEPress, Italy

Italian Journal of Medicine 2015; 9:77-81

doi:10.4081/itjm.2015.494 period the patient complained of acute abdominal and left side pain. Abdominal and pelvic computed tomography $(\mathrm{CT})$ scan showed right iliac artery and common femoral right artery, mesenteric superior artery and a left kidney artery dissection and kidney infarction (Figures 1-3). The vascular surgeon prescribed conservative treatment, unfractionated heparin i.v. was started and the patient was transferred to our department. The patient's family history was relevant: she told us her father had died at age 50 from vascular complications following a splenectomy and her brother had died at age 26 from extended thoracic and abdominal aortic dissection. No other family event was reported at admission.

The patient has a history of varices treated three times with varicectomy, and she underwent mitral valvuloplasty for mitral insufficiency at age 32 complicated by deep vein thrombosis in the post-operative period. On physical examination patient skin was thin with visible veins, thin face and thin lips, exophthalmos, scarce subcutaneous fat, acrogeria, and hypermobility of small joints. The patient was hemodynamically stable, chest and abdomen examination revealed no abnormalities, and there was no significant pulse alteration. We hypothesized an inherited connective-tissue disorder and evaluated other possible vascular damage: a chest $\mathrm{CT}$ scan excluded thoracic aortic dissection or aneurism and significant alteration of coronary artery; an echocardiogram revealed a moderate mitral valve insufficiency and thickening of the mitral valve; carotid Doppler ultrasound excluded carotid, vertebral and subclavian dissection; and a brain CT scan excluded intracranial vascular diseases. After a few days of bed rest we authorized for the patient a restricted mobility limited to the use of the bathroom, and switched anticoagulation from unfractionated he- 
parin i.v. to low molecular weight heparin (LMWH) s.c. After 10 days a Doppler ultrasound confirmed right iliac and common femoral artery dissection with intimal flap (Figure 4) without dissection progression, significant artery obstruction or flow-alteration.

Our patient met the diagnostic criteria of Villefranche $^{1}$ (Table 1) for EDS vascular type. To confirm the diagnosis we searched for the specific mutation in the COL3A1 gene (a blood sample was sent to another institute for analysis). After two weeks the patient was discharged with instructions to continue LMWH and to avoid intense and moderate exertion. We planned a Doppler ultrasound after two weeks that showed intimal flap resolution, so LMWH was stopped and aspirin (100 $\mathrm{mg} /$ die) was started. Two months later we received the

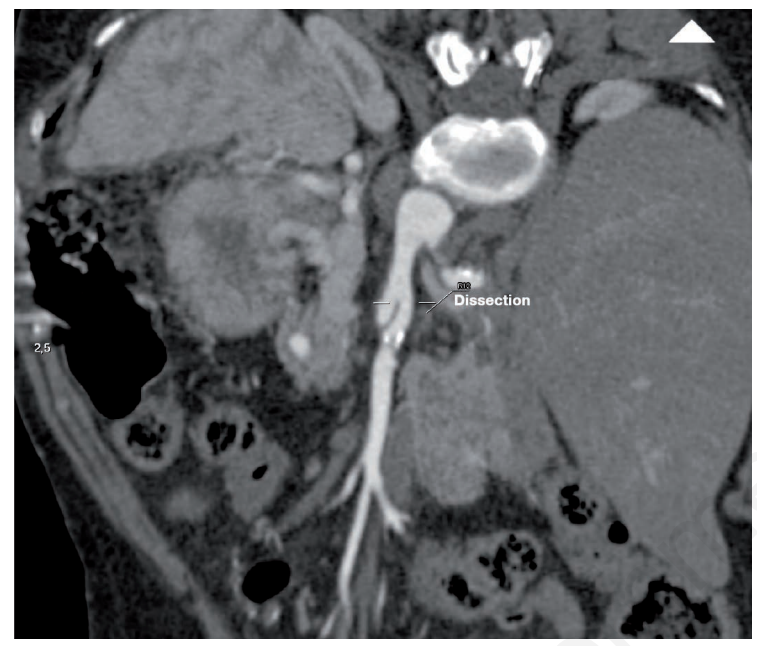

Figure 1. Computed tomography scan showing mesenteric superior artery dissection.

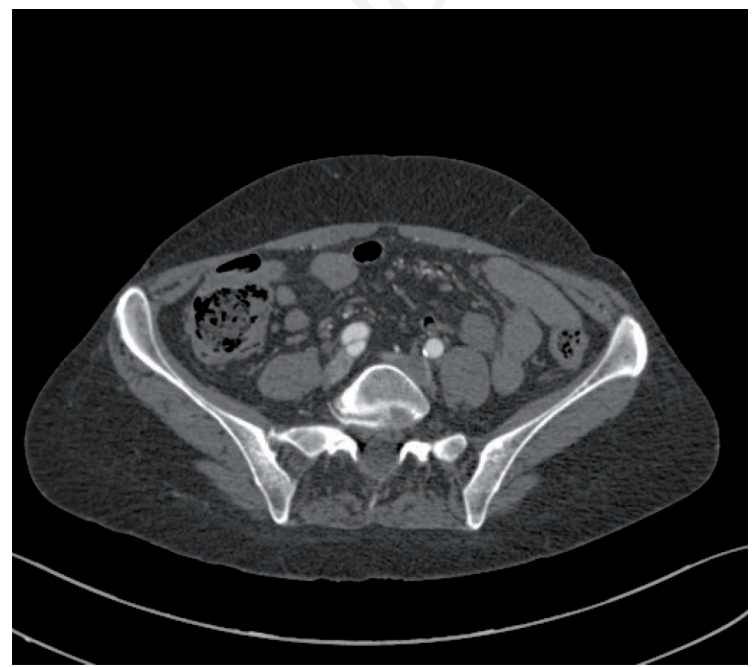

Figure 2. Computed tomography scan showing right common iliac artery dissection. gene molecular analysis results confirming the diagnosis of EDS type IV (vascular type). On the basis of recent randomized clinical trials, ${ }^{4}$ we started celiprolol therapy (200 mg/die). No adverse effects were observed, and we contacted the patient's family members requesting extended genetic analysis.

The complete family history is characterized by major vascular events. We did not obtain data on the patient's grandfather and grandmother. The obtained data confirm the patient's father died from aortic dissection while the mother is still alive and healthy. The patient had three sisters, three brothers, and two daughters. We reviewed clinical data present in our hospital and discovered that a sister died at age 15 having a congenital heart disease with Eisenmenger's syndrome. Further, a

Table 1. Villefranche diagnostic criteria for vascular type of Ehlers-Danlos syndrome.

\begin{tabular}{ll}
\hline Major diagnostic criteria & Minor diagnostic criteria \\
\hline Thin, translucent skin & Acrogeria \\
\hline $\begin{array}{l}\text { Arterial/intestinal/uterine } \\
\text { fragility or rupture }\end{array}$ & Hypermobility of small joints \\
\hline Extensive bruising & Tendon and muscle rupture \\
\hline Characteristic facial & Talipes equinovarus (clubfoot) \\
& appearance \\
& Early-onset varicose veins \\
& Arteriovenous, carotid-cavernous \\
& sinus fistula \\
& Pneumothorax/pneumohemothorax \\
& Gingival recession \\
& Positive family history, sudden death \\
& in (a) close relative(s) \\
\hline
\end{tabular}

The presence of any two or more of the major criteria is highly indicative of the diagnosis, and laboratory testing is strongly recommended. Modified from Beighton et al., 1998. ${ }^{1}$

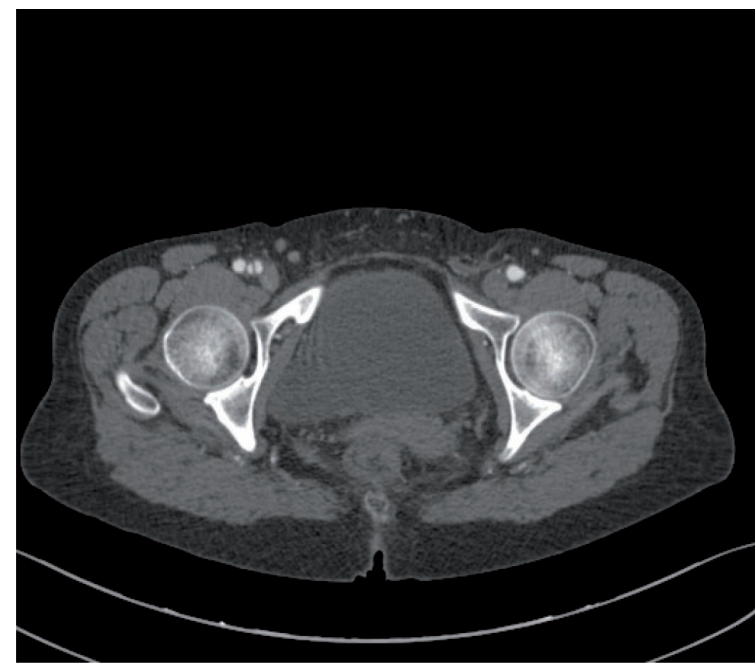

Figure 3. Computed tomography scan showing right common femoral artery dissection. 
brother died at age 26 in our hospital after an aorticfemoral by-pass complicated in the post-operative phase by thoracic aortic dissection. In the last three months of his life he had retroperitoneal hematoma after rupture of a right renal artery aneurism (endovascular treatment). This event was complicated by massive deep vein thrombosis treated with caval filter (recent retroperitoneal bleeding); he was studied by angiography that revealed right subclavian aneurism and right iliac dissection treated with endovascular stenting.

We contacted all the living brothers and sisters and the two daughters, and we performed genetic analyses of the COL $3 \mathrm{Al}$ gene, as well as clinical and instrumental examinations of the patients.

One sister, one brother and one of the two daughters expressed the COL3A1 mutation; they had the typical facial features, varix, hypermobility of small joints and easy bruising. The two women had a history of obstetric complication (post-partum bleeding, uterine rupture), and the brother had a history of recurrent shoulder dislocation. We performed a complete vascular screening of these three patients with EDS IV type with carotid, aortic and periphery ultrasound that excluded vascular problems. We found no significant alteration at the echocardiogram.

Our patient, her sister and her daughter were referred to another center specialized in rare diseases and EDS for long-term follow-up and genetic counseling (IRCCS Foundation Ca' Granda - Major Policlinic Hospital of Milan, Occupational Medicine - Rare Diseases Elhers Danlos syndrome).

\section{Follow-up}

One year later the patient made a routine Doppler ultrasound that showed an asymptomatic dissection of the right superficial femoral artery (Figure 5). A CT scan confirmed the data and showed also a focal dissection of the left common iliac artery. The patient was prudently hospitalized in our department and mobility was restricted to use of bathroom for a week. We had no reason to consider these dissections acute so we did not treat the patient with heparin; the vascular surgeon confirmed this conservative approach. After a week no progression of the dissection appeared in Doppler ultrasound and CT scan. We increased celiprolol therapy, in agreement with EDS centre, from 200 to $300 \mathrm{mg} / \mathrm{die}$ and the patient was discharged with close clinical and instrumental follow-up. Today the patient is 54 years old, no other vascular events have occurred, and the continued celiprolol treatment is still working with a good quality of life.

\section{Discussion}

The clinical diagnosis of Ehlers-Danlos syndrome type IV, the vascular type, rests on the finding of at least two of four clinical criteria: easy bruising; thin skin with visible veins; characteristic facial features; and rupture of arteries, uterus, or intestines. The diagnosis is confirmed by the demonstration that cultured fibroblasts synthesize abnormal type III pro-collagen molecules or by the identification of a mutation in the gene for type III pro-collagen (COL3A1). ${ }^{5}$ Our patient had all the four major criteria for Ehlers-Danlos syndrome type IV diagnosis and the specific mutation (heterozygous missense germinal mutation c. $2203 \mathrm{G}>\mathrm{A}$ - p.(Gly735Arg) - in exon 32 of COL $3 \mathrm{Al}$ gene). This syndrome is an autosomal dominant disorder, with variable penetrance, affecting approximately 1 in 100,000 to 250,000 people. $^{2}$ In part because of its rarity, the diagnosis is often made only after a catastrophic complication. In fact our patient, despite a significant family history, had a late diagno-

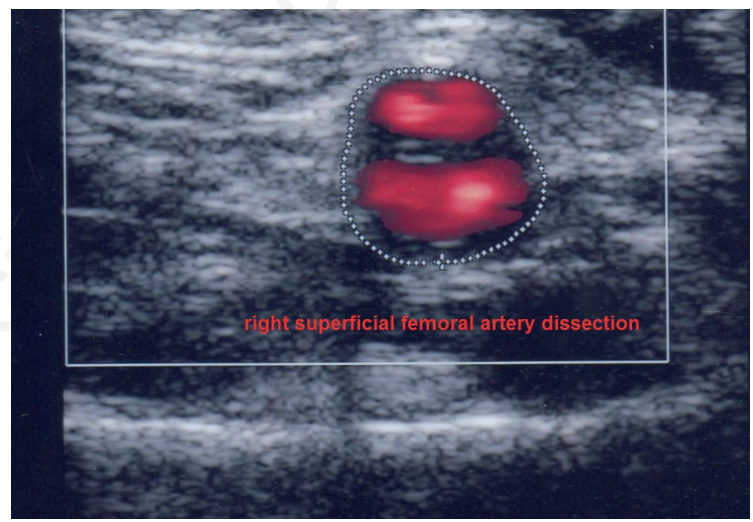

Figure 4. Doppler ultrasound showing right external iliac artery dissection in a transversal section.

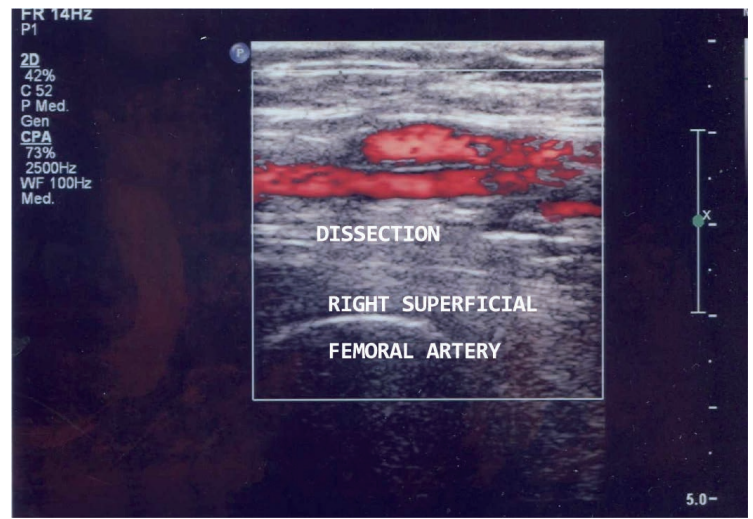

Figure 5. Doppler ultrasound (longitudinal section) showing right superficial femoral artery dissection with presence of double-lumen. 
sis of EDS after an artery dissection and unfortunately her brother died at a young age after several vascular complications. Patients frequently die in their third and fourth decade and survival beyond 50 years is exceptional. ${ }^{3}$ Arterial rupture is the most common cause of sudden death ${ }^{1}$ so we can say that our patient has now reached an age above average even in the presence of a significant history of arterial complications.

We tested all living brothers and sisters and the two daughters. Half of them, according to dominant disorder, had COL3A1 mutation. None of them, unlike our patient and her deceased brother, had a history of vascular events in agreement with the possibility of different genetic penetrance of EDS. The sister and the daughter with EDS had a history of obstetric complications. This finding is typical of EDS vascular type in which there is a high risk of maternal mortality, high incidence of spontaneous abortion, and uterine rupture and pre- and postpartum arterial bleeding. ${ }^{1}$ Rupture of the large arteries in EDS is peculiar to the type IV variety. Multiple arterial dissection diagnosis in our patient was made by angio-CT scan and follow-up was done with Doppler ultrasound and CT scan. Several authors emphasized the importance of the choice of diagnostic methods: arteriography is associated with a high complication rate in EDS patients including hematoma formation, arterial disruption, flaps and false aneurysms. The use of non-invasive diagnostic techniques such as duplex scan, CT and magnetic resonance imaging scans, and intravenous arteriography is described and should be used to investigate vascular problems in these patients. Angiography should be used only if it is possible to modify the therapeutic management. ${ }^{6}$ There is some debate about the best therapeutic approach to vascular complications in EDS patients. In the current case, after discussion with the vascular surgeon, a conservative approach was adopted. After seven days of bed rest we made gradual mobilization of the patient with no progression of symptoms, with no dissection progression in instrumental evaluation, and with no short-term complications. Our patient had an iliac and femoral artery dissection recurrence one year later, but the same approach was successfully adopted.

With regard to treatment of vascular complications in vascular EDS, there seems to be a consensus of opinion for non-operative management whenever possible because of elevated rates of intraoperative and postoperative complications in this population, ${ }^{7}$ with operative management reserved for patients in urgent and lifethreatening conditions. Some reports have estimated mortality to be between $20 \%$ and $65 \%$ in patients with vascular Ehlers-Danlos syndrome who undergo vascular reconstruction.

A recent study suggests that elective surgical management of vascular complications can be associated with good outcomes and should not be withheld until rupture or acute symptoms arise. ${ }^{8}$ For these authors EDS patients can safely undergo elective operations, including both endovascular and open vascular repair procedures, with a multidisciplinary approach to patient evaluation (geneticist, anesthetist, vascular surgeon) and with use of adjunctive techniques to reduce operative trauma in the open and endovascular setting. A recent consensus document indicates that stent-graft therapy should be avoided in vascular Ehlers-Danlos syndrome patients and in those with other connective tissue diseases. ${ }^{9}$ There is no specific therapy for the alteration of collagen production in EDS patients. Some authors have suggested the use of ascorbic acid which stimulates the synthesis of collagen, ${ }^{10}$ but until now no treatment has been proven to prevent clinical events. Only recently data have been published that prove the effect of celiprolol in the prevention of cardiovascular events in patients with vascular EDS. The study demonstrates a threefold reduction in arterial events in patients treated with celiprolol compared with those who received no treatment. ${ }^{4}$ Patients with vascular Ehlers-Danlos syndrome have decreased intima-media thickness that is associated with an increased risk of arterial dissection or rupture. Ong et al. ${ }^{4}$ noted that common carotid artery stiffness increased in response to celiprolol; chronic $\beta 2$ stimulation might enhance collagen synthesis through increased expression of TGF $\beta$. Thus, in response to celiprolol, an upregulation of collagen synthesis might have strengthened the arterial wall, reducing its susceptibility to rupture. On the basis of such evidence, after genetic analysis that confirmed the diagnosis of EDS, we started celiprolol therapy ( $200 \mathrm{mg} / \mathrm{die})$. No adverse effects were observed, and one year later, after the recurrence of asymptomatic iliac artery dissection, we increased celiprolol to 300 $\mathrm{mg} / \mathrm{die}$. Even this dosage was well tolerated, and no major vascular events occurred to-date.

\section{Conclusions}

EDS type IV, a rare connective tissue disease, should always be suspected in young or middle age patients who present with vascular accident, spontaneous bowel perforation or obstetric complications. Family medical history and clinical examination should be obtained, searching for typical signs of the disease and, if found, skin fibroblast culture or genetic analysis of COL $3 \mathrm{Al}$ gene should be performed to definitely establish the diagnosis. After definitive diagnosis, relative's genetic evaluation and genetic counseling is recommended, because it allows a close follow-up of patients with EDS and proper management of the same in case of vascular events. Vascular accidents should be investigated with non-invasive modalities and conservative treatment should be used with operative management reserved in patient with urgent and life-threatening condition. 
If tolerated, chronic celiprolol treatment can be administered for prevention of cardiovascular events. A clinical and instrumental follow-up needs to be done regularly, if possible in a rare diseases specialized center.

This case demonstrates as a careful evaluation of clinical signs, clinical history of the patient and his family has allowed a definitive diagnosis, proper management of the patient during the acute event and in terms of prophylaxis of recurrence; this assessment has also been extended to the family unit and this could prevent or at least lead to an optimal management of any vascular or other catastrophic events.

\section{References}

1. Beighton P, De Paepe A, Steinmann B, et al. EhlersDanlos syndromes: revised nosology, Villefranche, 1997. Am J Med Genet 1998;77:31-7.

2. Pope FM, Nicholls AC, Jones PM, et al. EDS IV (acrogeria): new autosomal dominant and recessive types. J R Soc Med 1980;73:180-6.

3. Dwivedi AJ, Hamdallah O, Morris ME, et al. Varying presentations in patients with symptomatic type IV vas- cular Ehlers-Danlos syndrome. Vasc Endovasc Surg 2012;46:163-6.

4. Ong K-T, Perdu J, De Backer J, et al. Effect of celiprolol on prevention of cardiovascular events in vascular Ehlers-Danlos syndrome: a prospective randomised, open, blinded-endpoints trial. Lancet 2010;376:1476-84.

5. Pepin M, Schwarze U, Superti-Furga A, Byers PH. Clinical and genetic features of Ehlers-Danlos syndrome type IV, the vascular type. NEJM 2000;342:673-80.

6. Habib K, Memon MA, Reid DA, Fairbrother DJ. Spontaneous common iliac arteries rupture in Ehlers-Danlos syndrome type IV: report of two cases and review of the literature. Ann R Coll Surg Engl 2001;83:96-104.

7. Chu LC, Johnson PT, Dietz HC, et al. Vascular complications of Ehlers-Danlos syndrome: CT findings. AJR Am J Roentgenol 2012;198:482-7.

8. Brooke BS, Arnaoutakis GJ, McDonnell NB, Black III JH. Contemporary management of vascular complications associated with Ehlers-Danlos syndrome. J Vasc Surg 2010;51:131-9.

9. Svensson LG, Kouchoukos NT, Miller DC, et al. Expert consensus document on the treatment of descending thoracic aortic disease using endovascular grafts. Ann Thor Surg 2008; 85: S1-41.

10. Cikrit DF, Glover RJ, Dalsing MC, Silver DV. The Ehlers-Danlos specter revisited. Vasc Endovasc Surg 2000;36:213-7. 